\title{
Cortical Projections of Functionally Identified Thalamic Trigeminovascular Neurons: Implications for Migraine Headache and Its Associated Symptoms
}

\author{
Rodrigo Noseda, ${ }^{1}$ Moshe Jakubowski, ${ }^{1}$ Vanessa Kainz, ${ }^{1}$ David Borsook, ${ }^{2}$ and Rami Burstein ${ }^{1}$ \\ ${ }^{1}$ Department of Anesthesia and Critical Care, Beth Israel Deaconess Medical Center, Harvard Medical School, Boston, Massachusetts 02215, and 2Pain and \\ Analgesia Imaging and Neuroscience Group, Brain Imaging Center, Department of Psychiatry, McLean Hospital, Harvard Medical School, Belmont, \\ Massachusetts 02478
}

This study identifies massive axonal arbors of trigeminovascular (dura-sensitive) thalamic neurons in multiple cortical areas and proposes a novel framework for conceptualizing migraine headache and its associated symptoms. Individual dura-sensitive neurons identified and characterized electrophysiologically in first-order and higher-order relay thalamic nuclei were juxtacellularly filled with an anterograde tracer that labeled their cell bodies and processes. First-order neurons located in the ventral posteromedial nucleus projected mainly to trigeminal areas of primary (S1) as well as secondary (S2) somatosensory and insular cortices. Higher-order neurons located in the posterior (Po), lateral posterior (LP), and lateral dorsal (LD) nuclei projected to trigeminal and extra-trigeminal areas of S1 and S2, as well as parietal association, retrosplenial, auditory, ectorhinal, motor, and visual cortices. Axonal arbors spread at various densities across most layers of the different cortical areas. Such parallel network of thalamocortical projections may play different roles in the transmission of nociceptive signals from the meninges to the cortex. The findings that individual dura-sensitive Po, LP, and LD neurons project to many functionally distinct and anatomically remote cortical areas extend current thinking on projection patterns of high-order thalamic neurons and position them to relay nociceptive information directly rather than indirectly from one cortical area to another. Such extensive input to diverse cortical areas that are involved in regulation of affect, motor function, visual and auditory perception, spatial orientation, memory retrieval, and olfaction may explain some of the common disturbances in neurological functions during migraine.

\section{Introduction}

The headache phase of a migraine attack is thought to be mediated by the trigeminovascular pathway. The primary afferents of the pathway reside in the trigeminal ganglion, and their axons split into a peripheral branch innervating blood vessels in the cerebral dura and pia maters and a central branch projecting to the spinal trigeminal nucleus $(\mathrm{SpV})$. Cell bodies of second-order trigeminovascular neurons are found mainly in laminae I and $\mathrm{V}$ of the dorsal horn of the medulla and upper cervical (C1-C2) spinal segments (Strassman et al., 1994). Cell bodies of thirdorder trigeminovascular neurons are found mainly in the posterior (Po) and ventral posteromedial (VPM) thalamic nuclei.

Electrophysiological and anatomical studies have characterized the physiologic response properties of neurons along the trigeminovascular pathway. The primary afferents are activated by mechanical and chemical stimuli emanating from the dura

Received June 28, 2011; revised Aug. 5, 2011; accepted Aug. 10, 2011.

Author contributions: R.N., M.J., and R.B. designed research; R.N. and V.K. performed research; R.N., M.J., D.B., and R.B. analyzed data; M.J. and R.B. wrote the paper.

The authors declare no competing financial interests.

This research was supported by NIH Grants NS-051484 and NS-069847 (R.B.).

Correspondence should be addressed to Rami Burstein, CLS-649, 3 Blackfan Circle, Boston, MA 02215. E-mail: rburstei@bidmc.harvard.edu.

DOI:10.1523/JNEUROSCI.3285-11.2011

Copyright $\odot 2011$ the authors $\quad 0270-6474 / 11 / 3114204-14 \$ 15.00 / 0$
(Mayberg et al., 1981, 1984; Strassman et al., 1996; Levy and Strassman, 2002). Trigeminovascular SpV neurons process nociceptive signals from the dura together with various sensory signals they receive from the cornea, facial skin, and cephalic and neck muscles (Davis and Dostrovsky, 1988a; Kaube et al., 1992; Strassman et al., 1994; Angus-Leppan et al., 1997; Ebersberger et al., 1997; Burstein et al., 1998; Yamamura et al., 1999). Trigeminovascular thalamic neurons receive and process nociceptive signals from the dura together with various sensory signals from all somatic dermatomes (Davis and Dostrovsky, 1988b; Zagami and Lambert, 1990, 1991; Angus-Leppan et al., 1995; Shields and Goadsby, 2005; Burstein et al., 2010).

Anatomical mapping of axonal projections and termination sites of peripheral and central trigeminovascular neurons remains scarce because of technical limitations. Neuronal tract tracing from the meninges was found to label primary afferent soma in the trigeminal ganglion and their axonal processes in laminae I-IV of the upper cervical and medullary dorsal horn (Liu et al., 2004, 2008). Axonal projections of individual secondorder dura-sensitive neurons were traced electrophysiologically from laminae I and $\mathrm{V}$ of $\mathrm{SpV}$ to the midbrain, in which they bifurcated into one branch that projected to the Po, VPM, and parafascicular thalamic nuclei and a second branch that projected to the anterior, lateral, perifornical and posterior hypothalamic nuclei, and lateral preoptic area (Burstein et al., 1998). 
A
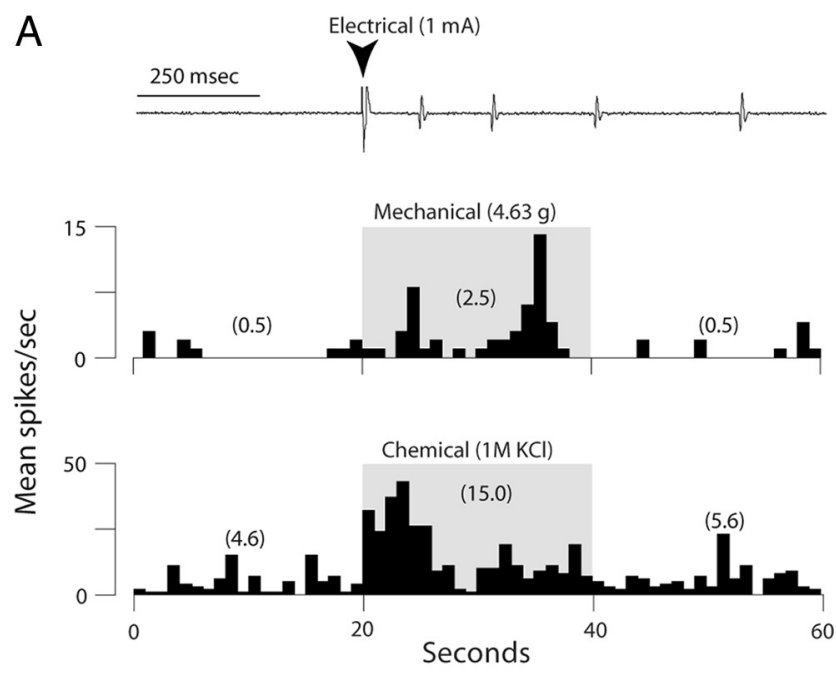

B Ophthalmic skin

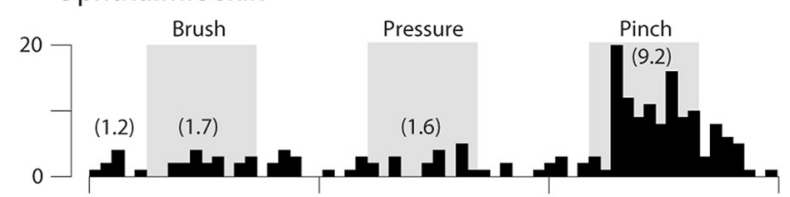

Ear

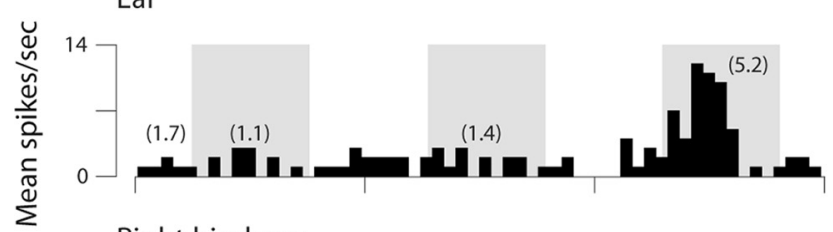

Right hindpaw

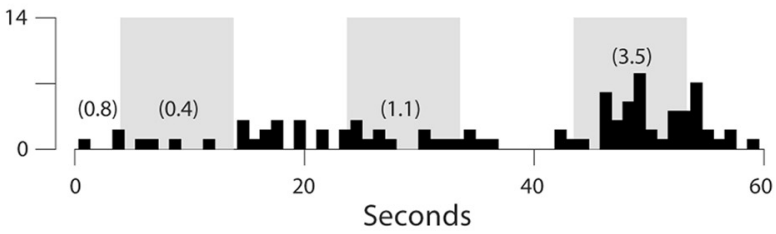

C

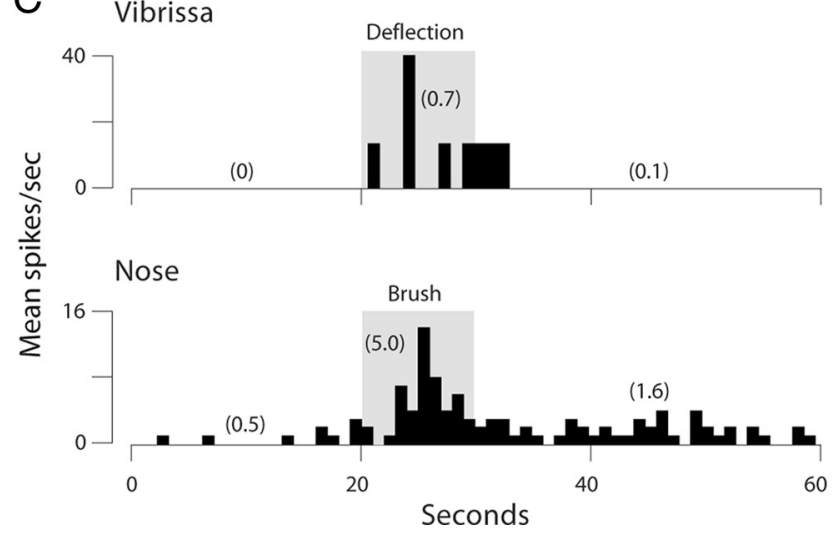

Figure 1. Identification and classification of trigeminovascular thalamic neurons. $A$, Individual dura-sensitive neurons exhibited discrete discharges in responses to electrical, mechanical, and chemical stimulation of the cranial dura overlying the transverse sinus. $\boldsymbol{B}$, Dura-sensitive neurons that responded to noxious stimulation of the skin (pinch) were classified as nociceptive if they exhibited no discharge in response to innocuous skin stimuli (brush, pressure). C, Durasensitive neurons that responded to gentle mechanical stimulation were classified as nonnociceptive. Stimulus intervals are indicated by gray areas. Numbers in parentheses are spikes per seconds for the corresponding intervals. Bin width, $1 \mathrm{~s}$.
The axonal projections and termination fields of trigeminovascular thalamic neurons remains essentially unexplored. In this study, we used a technique that combines single-unit recording with subsequent iontophoretic administration of an anterograde tracer into targeted trigeminovascular thalamic neurons, thus labeling individual cell bodies and their processes with exquisite details (Pinault, 1996; Gauriau and Bernard, 2004). Using this approach, we have successfully mapped the axonal trajectory and cortical terminations of three thalamic neurons that were characterized as receiving nociceptive input from the dura and photic signals from the retina (Noseda et al., 2010). The aim of the current study was to map and compare the cortical projections of trigeminovascular neurons located in first-order and higher-order relay thalamic nuclei, which are thought to play differential roles in thalamocorticothalamic processing of sensory information (Sherman, 2005; Jones, 2009).

\section{Materials and Methods}

Animals and surgical procedures. Experiments were approved by the Beth Israel Deaconess Medical Center and Harvard Medical School standing committees on animal care and were conducted in accordance with the National Institutes of Health Guide for the Care and Use of Laboratory Animals. Male Sprague Dawley rats (250-350 g) were initially anesthetized with methohexital sodium ( $45 \mathrm{mg} / \mathrm{kg}$, i.p.) for endotracheal intubation and cannulation of the right femoral vein. Each rat was then mounted on a stereotaxic frame and switched to inhalation anesthesia: a mixture of isoflurane and pure oxygen delivered at a rate of $100 \mathrm{ml} / \mathrm{min}$. Craniotomies (see below) were performed using $2.5 \%$ isoflurane, whereas the subsequent experimental procedure was performed under $1-1.2 \%$ isoflurane. End-tidal $\mathrm{CO}_{2}$, blood oxygen saturation, as well as breathing and heart rates, were monitored and kept within physiological values throughout the experiment. Core body temperature was maintained at $37^{\circ} \mathrm{C}$ using a heating blanket. To immobilize the rats during the experiment, a mixture of the muscle relaxants vecuronium and pancuronium (each $1 \mathrm{mg} / \mathrm{ml}$ in lactated Ringer's solution) was infused continuously via the intravenous cannula at a rate of $0.3 \mathrm{mg} \cdot \mathrm{kg}^{-1} \cdot \mathrm{h}^{-1}$.

Two craniotomies were performed. A $4 \times 4 \mathrm{~mm}$ craniotomy was made at the lambdoid suture on the left side of the skull to allow placement of a stimulating electrode on the dura overlying the transverse sinus. A $2 \times$ $2 \mathrm{~mm}$ craniotomy was made on the right side of the skull $(2.5 \mathrm{~mm}$ behind the coronal suture; $2.5 \mathrm{~mm}$ lateral to the midline) to access the thalamus with a micropipette that was used for single-unit recording and juxtacellular administration of an anterograde tracer into the target cell. The exposed dura was kept moist using a modified synthetic interstitial fluid (in mM: $135 \mathrm{NaCl}, 5 \mathrm{KCl}, 1 \mathrm{MgCl}_{2}, 5 \mathrm{CaCl}_{2}, 10$ glucose, and 10 HEPES, $\mathrm{pH} 7.2$ ).

Single-unit recordings. A glass micropipette (20-30 $\mathrm{M} \Omega$ impedance) was lowered into the right posterior thalamus in search of single-unit discharges invoked by electrical pulses $(0.8 \mathrm{~ms}, 0.5-3.0 \mathrm{~mA}, 1 \mathrm{~Hz})$ at the exposed contralateral dura. A neuron was identified as dura sensitive if it exhibited discrete firing bouts in response to such electrical pulses, as well as mechanical (calibrated von Frey monofilament) and chemical $(1 \mathrm{M}$ $\mathrm{KCl}$ ) stimulation of the dura (Fig. 1A). A neuron that failed to respond to electrical, mechanical, and chemical stimulation of any part of the exposed area of the dura was referred to as dura insensitive. Whether or not such neurons were actually dura insensitive could not be determined with certainty because it was technically impossible to test them for stimulation of the entire area of the cerebral dura. All neurons were further classified as nociceptive or non-nociceptive based on their somatosensitivity to innocuous (brush) and noxious (pinch) mechanical stimulation of cephalic and extracephalic skin (Fig. $1 B, C$ ). Real-time waveform discriminator was used to create and store a template for the action potential evoked in the neuron under study by electrical pulses on the dura; spikes of activity matching the template waveform were acquired and analyzed online and offline using Spike 2 software (Cambridge Electronics Design).

Juxtacellular iontophoresis. Once characterized electrophysiologically, each neuron was infiltrated with the anterograde tracer that was delivered 
VPM
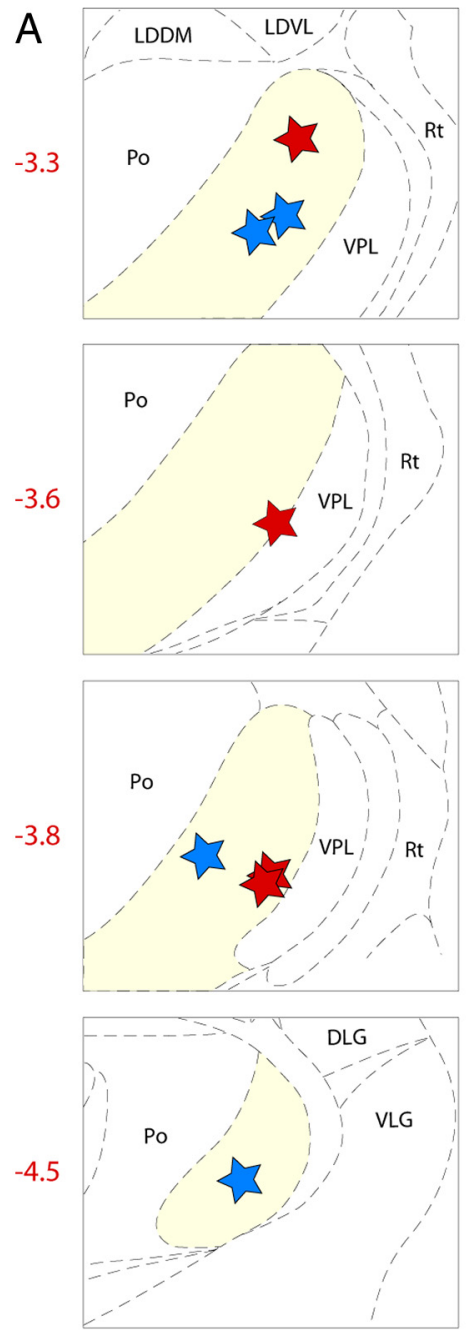

Po
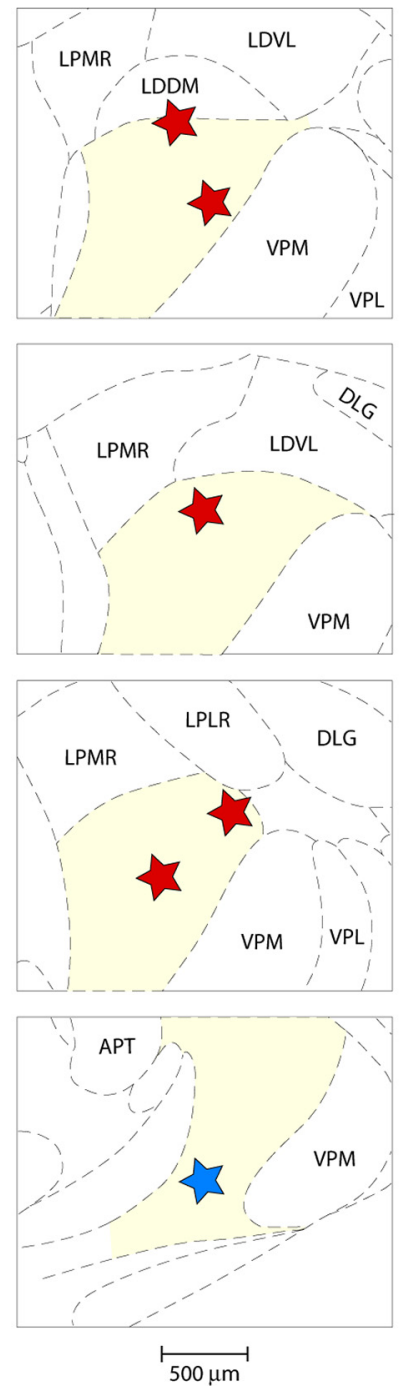

LD/LP
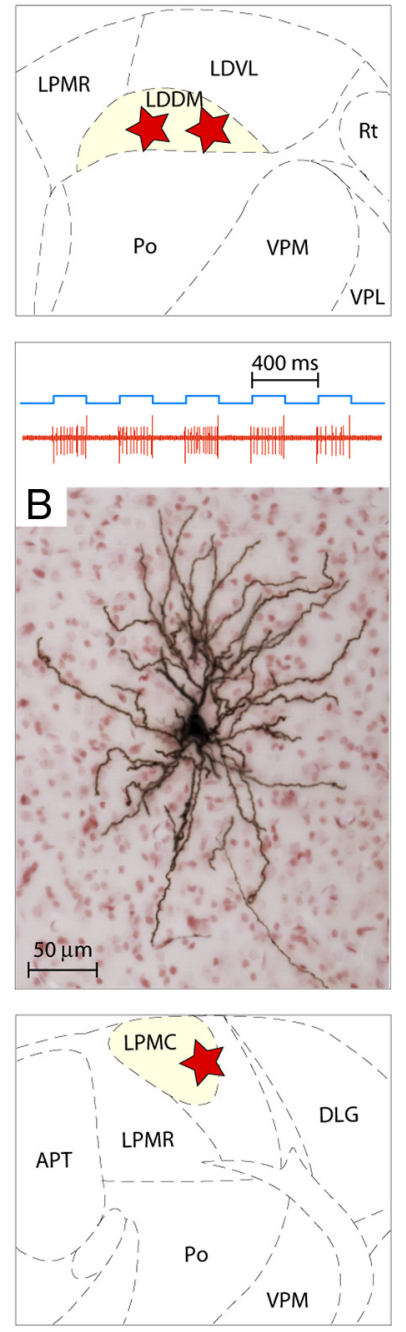

Figure 2. Localization of dura-sensitive (red stars) and dura-insensitive (blue stars) neurons in the thalamus. $A$, Localization of the cell bodies in the VPM, P0, and LD and LP nuclei (each nucleus highlighted in yellow). Numbers in red indicate the coronal plane (millimeters from bregma) according to the rat brain atlas (Paxinos and Watson, 1998). B, A photomicrograph of the cell body and dendritic tree of a dura-sensitive (LP) neuron that was labeled with the anterograde tracer TMR-dextran. Uptake of the tracer by the target cell body was achieved by inducing bouts of neuronal firing (red trace) in response to repetitive electrical pulses (blue trace) delivered from the recording micropipette, which was preloaded with the tracer. APT, Anterior pretectal nucleus; DLG, dorsal lateral geniculate nucleus; LDDM, laterodorsal thalamic nucleus, dorsomedial; LDVL, laterodorsal thalamic nucleus, ventrolateral; LPMR, lateral posterior thalamic nucleus, mediorostral; Po, posterior thalamic nuclear group; Rt, reticular thalamic nucleus; VPL, ventral posterolateral thalamic nucleus; VPM, ventral posteromedial thalamic nucleus.

iontophoretically from the recording micropipette as described previously (Pinault, 1996). The recording micropipette was preloaded with 3\% tetramethylrhodamine (TMR)-dextran (3000 molecular weight, anionic, lysine fixable; D-3308; Invitrogen), and the tracer was administered into the target cell body using a computer-controlled microelectrode amplifier (Axoclamp 900A; Molecular Devices). Uptake of the tracer by the cell body was achieved by inducing bouts of neuronal firing in response to positive current pulses (1-10 nA) that were delivered repetitively (200 $\mathrm{ms}$ on/off intervals) over a period of 10-60 min (Pinault, 1996; Fig. 2 B). At the end of the injection, infusion of muscle relaxants was discontinued, wounds were stitched and disinfected, the cannula was removed from the femoral vein, isoflurane concentration was reduced gradually, the endotracheal tube was removed, and the rat was allowed to resume spontaneous breathing under $>95 \%$ oxygen saturation.

Tissue processing. After a survival period of 3 or $4 \mathrm{~d}$, each rat was injected with an overdose of pentobarbital sodium $(100 \mathrm{mg} / \mathrm{kg}$, i.p.) and received intracardiac perfusion of $200 \mathrm{ml}$ of heparinized saline, followed by $500 \mathrm{ml}$ of a fixative solution consisting of $4 \%$ paraformaldehyde, $0.05 \%$ picric acid, $0.1 \mathrm{~m}$ PBS. Brains were extracted from the skull, soaked in the fixative solution for $2 \mathrm{~h}$, and cryoprotected in $30 \%$ sucrose phosphate buffer for $48 \mathrm{~h}$. Brains were then frozen on dry ice, mounted onto a cryostat (Leica), and cut into serial coronal sections ( $80 \mu \mathrm{m}$ thick) that were immersed in PBS using multiwell dishes. The wet sections were temporarily mounted on glass slides for a preliminary microscopic visualization of TMR-dextran fluorescence (excitation, $551 \mathrm{~nm}$; emission, $624 \mathrm{~nm}$; Leica). Sections containing the injection site were examined first to verify successful labeling of a target cell body and its dendrites. If so, additional sections were mounted and inspected for the presence of axonal branches anywhere in the cortex, and, if so, all sections ( $\sim 175$ per brain) were immersed back in PBS and immunostained (see below) for bright-field microscopy (Leica).

Immunohistochemistry. Free-floating sections were incubated on a rotating platform in the following sequence: (1) pretreatment with 3:1 methanol/PBS solution containing $1 \% \mathrm{H}_{2} \mathrm{O}_{2}$ to quench endogenous peroxidase activity ( $80 \mathrm{~min}$, room temperature); (2) preincubation with PBS solution containing $2 \%$ fetal bovine serum albumin and $1 \%$ Triton X-100 to block nonspecific binding of the primary antibody ( $1 \mathrm{~h}$, room temperature); (3) incubation with the primary anti- 


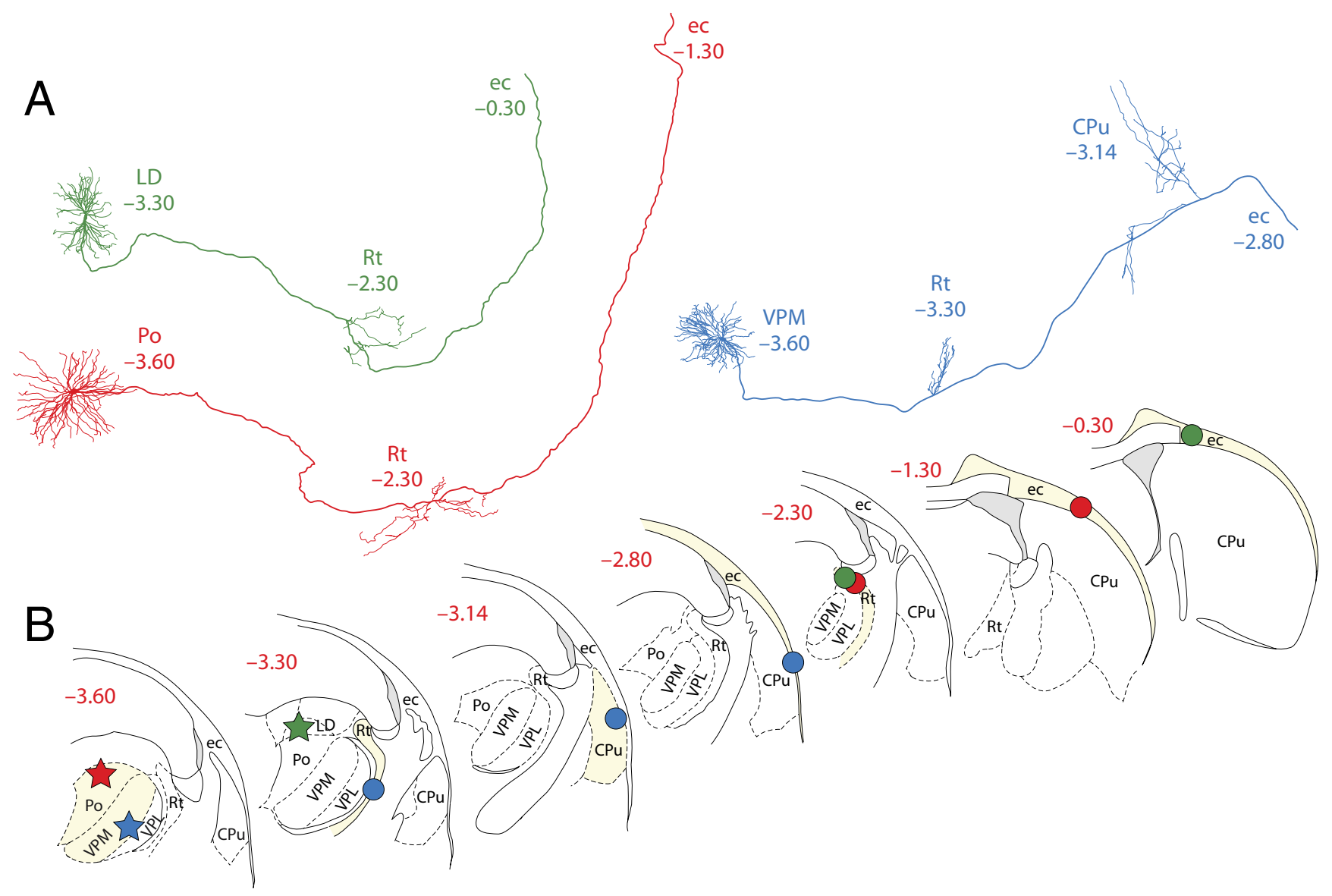

Figure 3. Subcortical mapping of axonal projections and their collaterals. $A$, Two-dimensional camera lucida reconstruction of cell bodies and dendritic trees of three dura-sensitive thalamocortical neurons, their collateral arbors in the reticular thalamic nucleus (Rt), and caudate-putamen ( $\mathrm{CPu}$ ), and their entry point in the external capsule (ec). $\boldsymbol{B}$, Localization of the cell bodies (stars) and landmarks of axonal trajectories (circles) of the neurons reconstructed above. Numbers in $\boldsymbol{A}$ and $\boldsymbol{B}$ indicate the coronal plane (millimeters from bregma). Color coding is the same in $\boldsymbol{A}$ and $\boldsymbol{B}$. For additional abbreviations, see Figure 2 .

body, rabbit anti-TMR (A-6397; Invitrogen), diluted 1:3000 in the preincubation solution $\left(48 \mathrm{~h}, 4^{\circ} \mathrm{C}\right)$; (4) incubation with the secondary antibody, biotinylated goat anti-rabbit (Jackson ImmunoResearch), diluted 1:500 in PBS (2 h, room temperature); and (5) signal amplification and detection using avidin-biotin complex (Vector Laboratories) and nickel-enhanced staining with 3,3'-diaminobenzidine. Sections were washed three times in $0.1 \mathrm{M}$ PBS between each of the above steps. Consecutive immunostained sections were then mounted in order on glass slides and coverslipped with Cytoseal.

Mapping axonal projections. The cell body and processes of each labeled neuron were traced and reconstructed in aggregate using bright-field microscopy (200× magnification) and camera lucida technique (Olympus). Reconstruction started with sections containing the labeled cell body and its dendritic tree and continued from one section to the next along the trajectory of the parent axon and its subcortical and cortical termination fields, defined as collateral branches that bifurcated from the parent axon. Digital images of the cell body, dendrites, and axonal termination fields were also documented using scanning microscopy compiled from 1 - to $1.5-\mu \mathrm{m}$ thick scans (Leica LAS $z$-stacking software).

For detailed histological analysis of neuroanatomical structures and cortical lamination, the mounted sections were stripped from the coverslips, counterstained with neutral red, and coverslipped again with Cytoseal. Digital images captured with scanning microscopy were used to mark the precise localization of the cell bodies of the labeled neurons and identify the cortical areas and laminae at their termination fields. Anatomical analysis was based on an atlas and a textbook of the rat brain (Vogt and Peters, 1981; Paxinos and Watson, 1998; DeFelipe et al., 2002; Shipp, 2007; Groh et al., 2010; Meyer et al., 2010).

\section{Results}

Using a glass micropipette preloaded with the anterograde tracer TMR-dextran, we isolated dura-sensitive and dura-insensitive neurons in several thalamic nuclei and attempted to inject the tracer juxtacellularly into each of the target cell bodies (one neuron per rat). Of 53 such attempts, 17 cases were included in the analysis, because they resulted in successful singular labeling of the target cell body and its axonal terminal arbors in the cortex. Two additional cases of successful labeling were excluded from the analysis, because they resulted in the labeling of two adjacent cell bodies, with no ability to determine which cell body and processes belonged to the target neuron. Of the remaining 34 attempts, 20 failed to produce any neuronal labeling, nine yielded labeling of cell body but no processes, and five resulted in labeling of the cell body and the proximal part of the parent axon without detectable labeling in the cortex.

\section{Neuronal classification, localization, and morphology}

Of the 17 labeled neurons included in the analysis, 12 were identified as dura sensitive (Fig. $1 A$; Fig. $2 A$, red stars) and 5 as dura insensitive (Fig. $2 A$, blue stars).

Based on their responses to innocuous and noxious skin stimuli, the dura-sensitive group included wide-dynamic range (WDR) units (four VPM, one Po), high-threshold (HT) units [three Po, two lateral dorsal (LD), one lateral posterior (LP)], and 


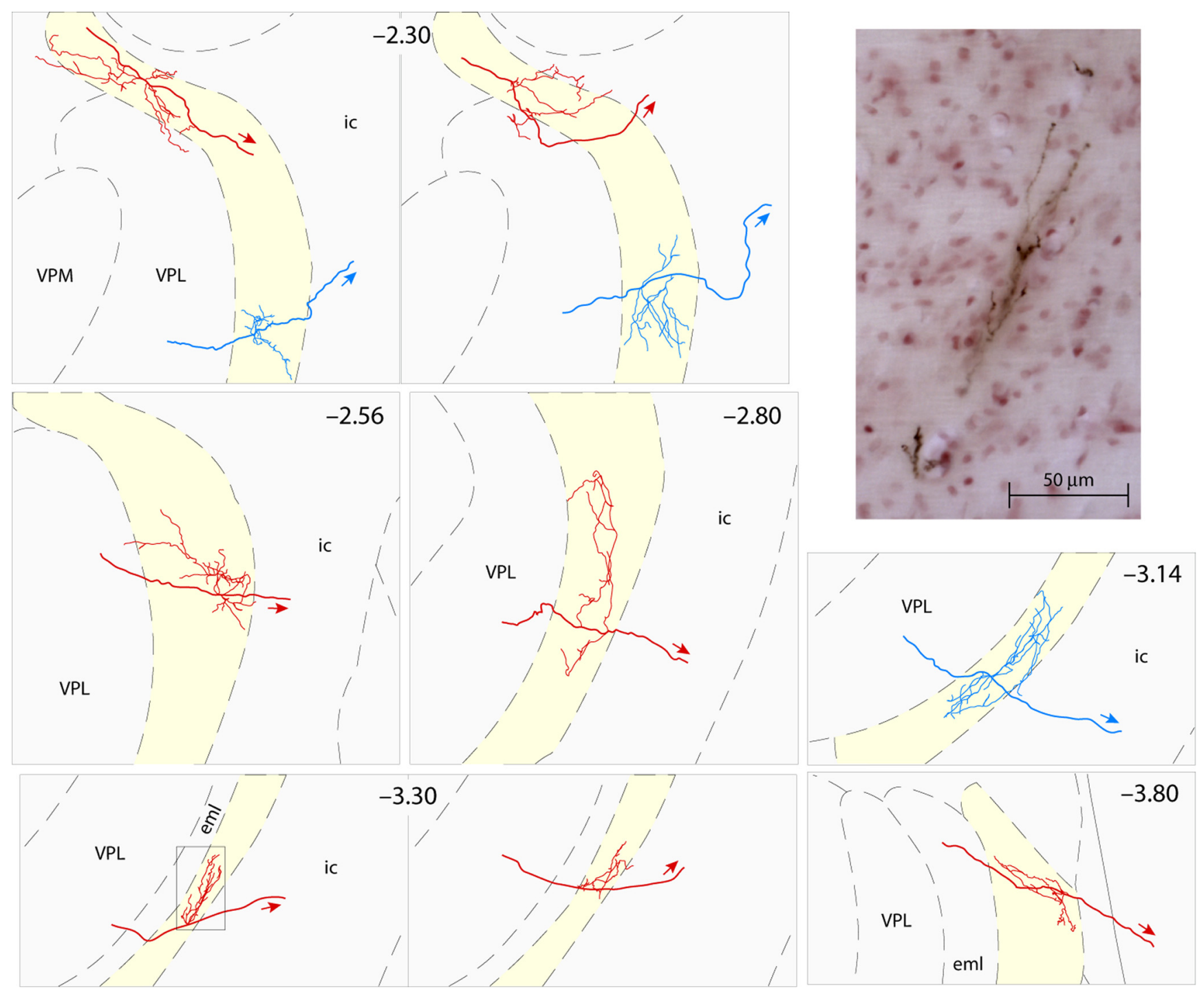

Figure 4. Reconstruction of individual axonal arbors in the reticular thalamic nucleus. The parent axons and their trajectories are indicated by arrows. Dura-sensitive neurons are red. Durainsensitive neurons are blue. The photomicrograph corresponds to the box in bottom left. Numbers indicate the coronal plane (millimeters from bregma). eml, External medullary lamina; ic, internal capsule. For additional abbreviations, see Figure 2.

an unclassified Po unit that exhibited no excitatory cutaneous receptive field.

The dura-insensitive group included a WDR VPM neuron, a low-threshold (LT) VPM neuron that responded to deflections of a single vibrissae, an LT VPM neuron responsive to ophthalmic skin brushing, a Po neuron whose activity was inhibited by noxious stimulation of facial or body skin, and an unclassified VPM neuron that exhibited no excitatory cutaneous receptive field.

The data below are dissected by neuronal identification (durasensitive vs dura-insensitive units) and by the thalamic localization of the cell bodies (VPM, Po, and LD/LP). Analysis by classification (nociceptive vs non-nociceptive) was not feasible, because only two neurons were non-nociceptive (LT).

Cell body size was similar between dura-sensitive and durainsensitive units and did not vary among the VPM, Po, or LD/LP nuclei. The maximal dendritic spread (Fig. $2 B$ ) was also similar between dura-sensitive and dura-insensitive neurons. However, the dendritic tree of VPM neurons $(263 \pm 12 \mu \mathrm{m})$ was significantly smaller compared with Po $(352 \pm 16)$ and LD/LP (344 \pm 32) neurons ( $p=0.0054$, Kruskal-Wallis test).

\section{Axonal trajectories from the thalamus to the cortex}

The cell bodies sampled in this study were located between 3.3 and $4.4 \mathrm{~mm}$ caudal to the plane of bregma (Fig. 2). The parent axons typically projected forward, laterally, and upward en route to the external capsule (Fig. 3), issuing prominent collateral arbors in the reticular (Rt) thalamic nucleus (Fig. 4). To identify the spatial organization of the subcortical axonal trajectories, we analyzed the relative positions of the cell body and axonal entry points in the Rt and external capsule. Linear regression analyses of 10 reconstructed neurons showed that the Rt arbor coordinates were correlated with the cell body position at the anteroposterior $\left(R^{2}=0.72, p=0.0020\right)$ and vertical $\left(R^{2}=0.66, p=\right.$ $0.0044)$ axes. The axonal entry points in the external capsule were, in turn, tightly correlated with the Rt arbor coordinates (anteroposterior, $R^{2}=0.75, p=0.0013$; vertical, $R^{2}=0.77, p=$ 0.0009 ; lateral, $R^{2}=0.73, p=0.0016$ ). Additional collateral arbors were issued in the caudate-putamen by 4 of the 17 neurons (Fig. 5). Of those, three were dura-sensitive VPM units classified as WDR; one was a dura-insensitive Po neuron whose activity was inhibited by noxious skin stimulation. 

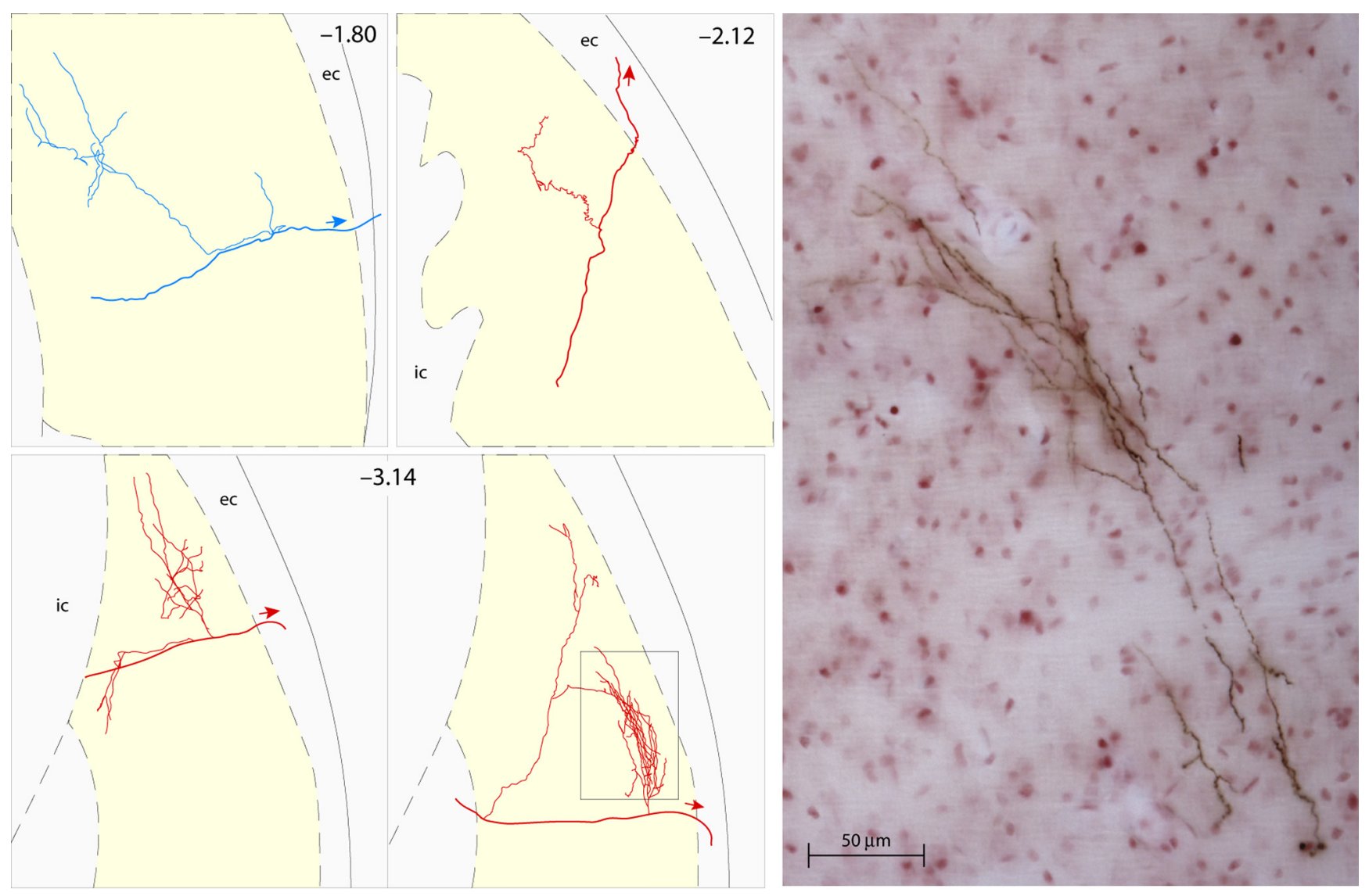

Figure 5. Reconstruction of individual axonal arbors in the caudate-putamen. The parent axons and their trajectories are indicated by arrows. Dura-sensitive neurons are red. Dura-insensitive neurons are blue. The photomicrograph corresponds to the box in bottom right. Numbers indicate the coronal plane (millimeters from bregma). ec, External capsule; ic, internal capsule.

\section{Cortical projections of dura-sensitive neurons}

VPM neurons $(\mathrm{n}=4)$

Dura-sensitive VPM neurons issued dense terminal arbors, which bifurcated from one or two branches of the parent axon, to the trigeminal barrel-field region of the primary somatosensory cortex (S1BF) and/or the secondary somatosensory cortex (S2). In addition, two of those neurons issued sparse collaterals in insular cortex, and a third neuron projected moderately into extra-trigeminal regions $S 1$ and sparsely into the primary and secondary motor cortices (M1/M2). Reconstruction of the cortical projections of one neuron is illustrated in Figure 6A.

Po neurons $(\mathrm{n}=5)$

Dura-sensitive Po neurons issued dense terminal arbors, which bifurcated from one or two branches of the parent axon, to S1BF and/or S2, as observed with their VPM counterparts. The Po units, however, also issued moderate-to-heavy projections to extra-trigeminal regions of $\mathrm{S} 1$ and to the primary auditory $(\mathrm{AuD} /$ Au1), retrosplenial (RSA), parietal association (PtA), and primary and secondary visual (V1/V2) cortices. One neuron projected heavily to M1/M2, as well. Reconstructions of the cortical projections of two neurons are illustrated in Figure 6B.

$L D / L P$ neurons $(\mathrm{n}=3)$

One dura-sensitive LD neuron projected exclusively and heavily to M1/M2 (Fig. 7A). A second LD neuron projected lightly to $\mathrm{M} 1 / \mathrm{M} 2$ and heavily to trigeminal and extra-trigeminal regions of S1. The one LP neuron in this category exhibited dense terminal arbors in V2, sparse fibers in V1, and moderate arbors in the ectorhinal cortex (Fig. 7B).

\section{Cortical projections of dura-insensitive neurons}

Like dura-sensitive cells, all dura-insensitive neurons, regardless of their somatosensory classification (two LT, two HT, one unclassified) issued terminal arbors in S1BF and/or S2. The one dura-insensitive neuron that issued additional terminals in the auditory and PtA cortices was nociceptive. Figure 8 shows terminal arbors that arise from two LT dura-insensitive neurons in $\operatorname{VPM}(A, B)$ and one Po neuron whose activity was inhibited by noxious skin stimuli $(C)$.

\section{Cortical layers of termination}

\section{Somatosensory cortices}

Terminal arbors were found in all layers of S1 and S2, with highest density in layers III and IV (Figs. 9-11). This general pattern was unrelated to thalamic localization of the cell body, dura sensitivity, or somatosensory classification.

\section{Insular cortex}

Layers III-VI of the insular cortex contained sparse-to-moderate density of labeled fibers arising from two dura-sensitive VPM neurons (Figs. 9, 12A); layers I-V were sparsely to moderately labeled by fibers arising from one dura-insensitive Po neuron (Fig. 9).

\section{Parietal association cortex}

Terminal fields in the PtA varied in density and layering patterns from neuron to neuron (Fig. 9): among three dura-sensitive Po neurons, one terminated sparsely in layers II and III (Fig. 12 B); a second terminated at various densities across layers I-V (most heavily in layers III-V); a third neuron projected lightly to mod- 

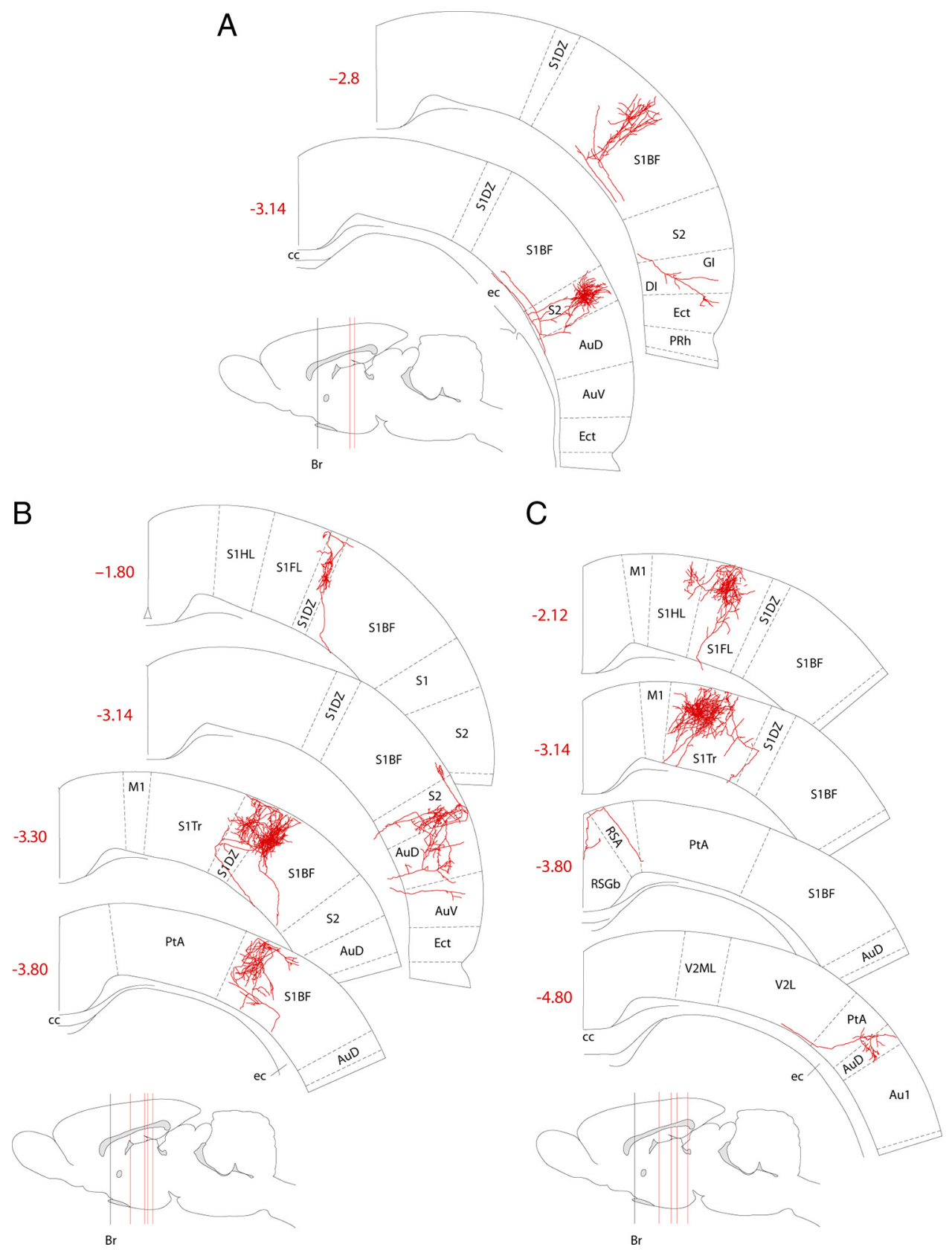

Figure 6. Reconstruction of cortical axonal arbors of individual dura-sensitive VPM $(\boldsymbol{A})$ and $\mathrm{P}_{0}(\boldsymbol{B}, \boldsymbol{C})$ neurons. Numbers in red indicate the coronal plane (millimeters from bregma). The levels of the different coronal drawings are indicated as red lines in the sagittal drawing of the brain. Note that VPM neurons project mainly to $S 1, S 2$, and the insula, whereas Po neurons project to multiple cortical areas outside the so-called pain matrix. Au1, Primary auditory cortex; AuD, secondary auditory cortex, dorsal; AuV, secondary auditory cortex, ventral; Br, bregma; cc, corpus callosum; DI, dysgranular insular cortex; Ect, ectorhinal cortex; Gl, granular insular cortex; M1, primary motor cortex; PtA, parietal association cortex; PRh, perirhinal cortex; RSA, retrosplenial cortex; RSGb, retrosplenial granular cortex, b region; S1, primary somatosensory cortex; S1BF, primary somatosensory cortex, barrel field; S1DZ, primary somatosensory cortex, dysgranular; S1FL, primary somatosensory cortex, forelimb; S1HL, primary somatosensory cortex, hindlimb; S1Tr, primary somatosensory cortex, trunk area; S2, secondary somatosensory cortex; V2L, secondary visual cortex, lateral area; V2ML, secondary visual cortex, mediolateral.

erately to layers II and III and heavily to layer V. One durainsensitive VPM neuron projected to all six layers of the PtA, most heavily to layers IV and V.

\section{Retrosplenial cortex}

In the RSA, layers I-III showed sparse-to-moderate density of fibers arising from four dura-sensitive Po neurons, with one of those neurons terminating densely in layer IV as well (Fig. 9).

Auditory and ectorhinal cortices

The AuD/Aul cortices contained sparse labeling in layers II and III (Fig. 12B). In the ectorhinal cortex, most dense labeling was observed in layers V and VI (Fig. 12C). Layer I was devoid of labeled fibers in both the auditory and ectorhinal cortices (Fig. 9).

M1/M2

Sparse projections to layers V and VI arose from a dura-sensitive VPM unit, moderate-to-heavy projections to layers II and III from a dura-sensitive Po unit, and light or heavy projections to layers I-III from two dura-sensitive LD units (Figs. 9, 12D).

Visual cortex

Labeling in the visual cortex was only observed with durasensitive neurons (Figs. 9, 13). Terminal arbors of moderate-to- 
A

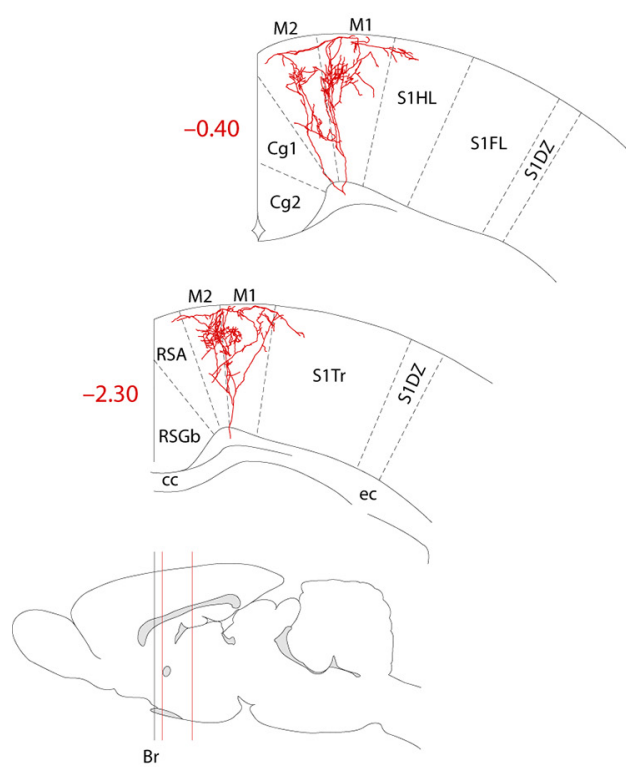

B

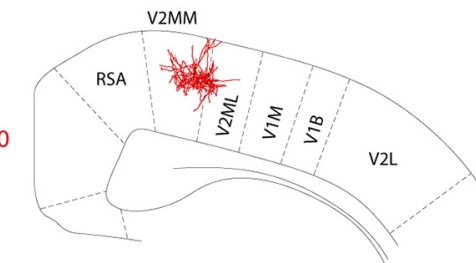

$-7.64$

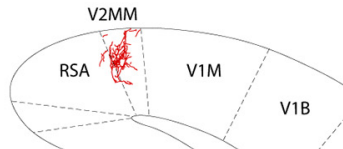

$-8.80$
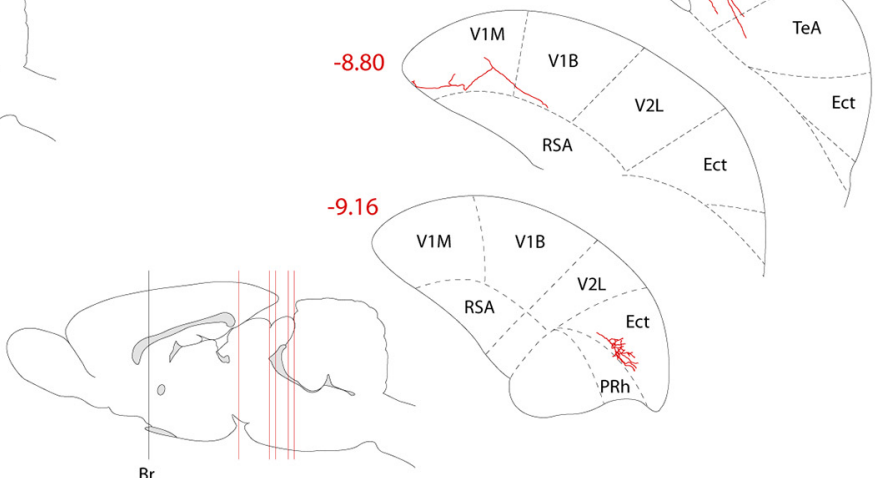

Figure 7. Reconstruction of cortical axonal arbors of individual dura-sensitive $L D(\boldsymbol{A})$ and $\operatorname{LP}(\boldsymbol{B})$ neurons. Note massive projections to visual and motor cortices. $\mathrm{Cg} 1$, Cingulated cortex, area 1; $\mathrm{Cg} 2$, cingulated cortex, area 2; M2, secondary motor cortex; V1B, primary visual cortex, binocular; V1M, primary visual cortex, monocular; V2MM, secondary visual cortex, mediomedial; TeA, temporal association cortex. For additional information and abbreviations, see Figure 6.

A

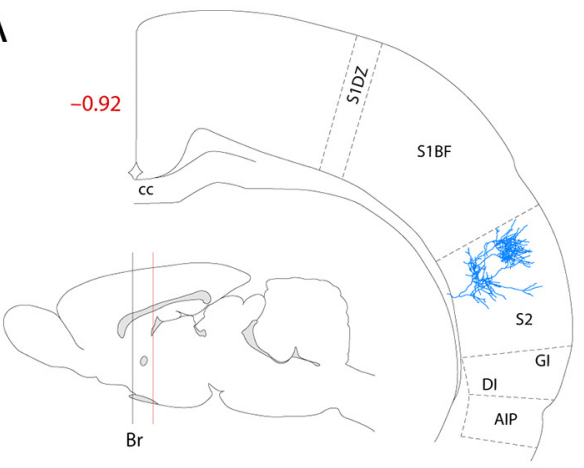

B

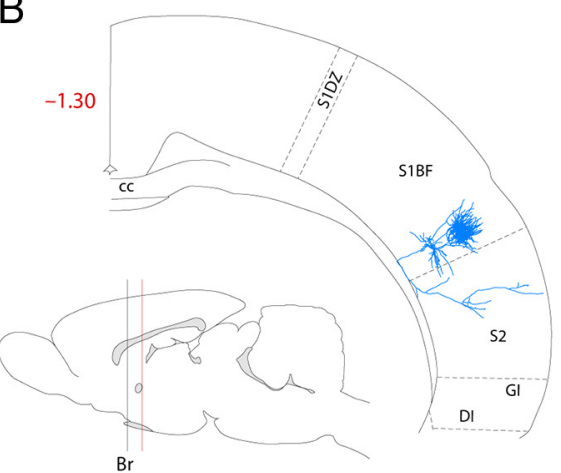

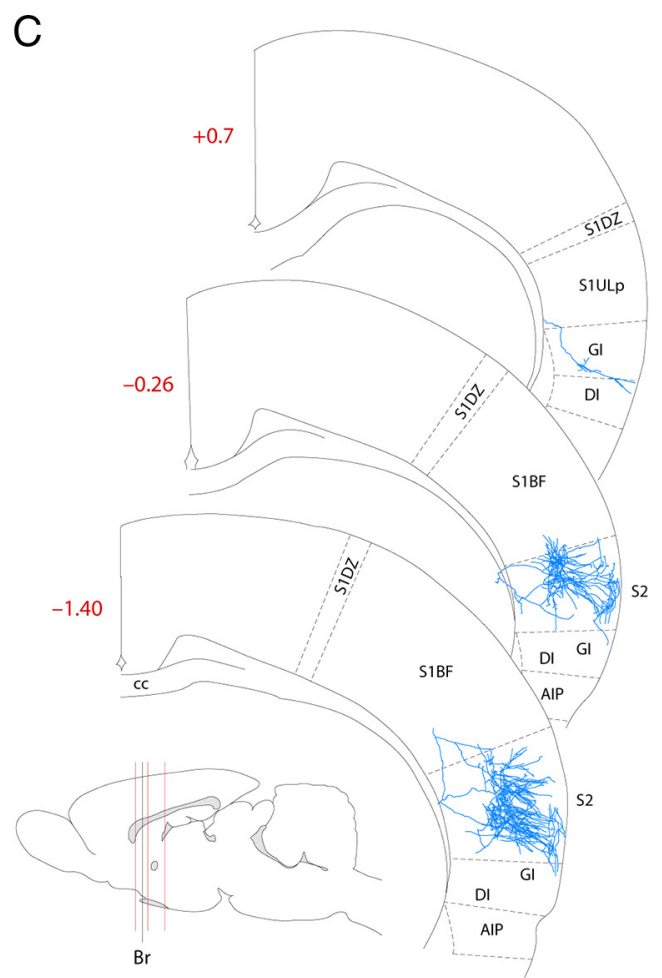

Figure 8. Reconstruction of cortical axonal arbors of individual dura-insensitive VPM $(A, B)$ and Po $(C)$ neurons. Note that most terminal arborization was restricted to cortical areas that process somatosensory information. AIP, Agranular insular cortex, posterior; S1ULp, primary somatosensory cortex, upper lip. For additional information, see Figure 6. 


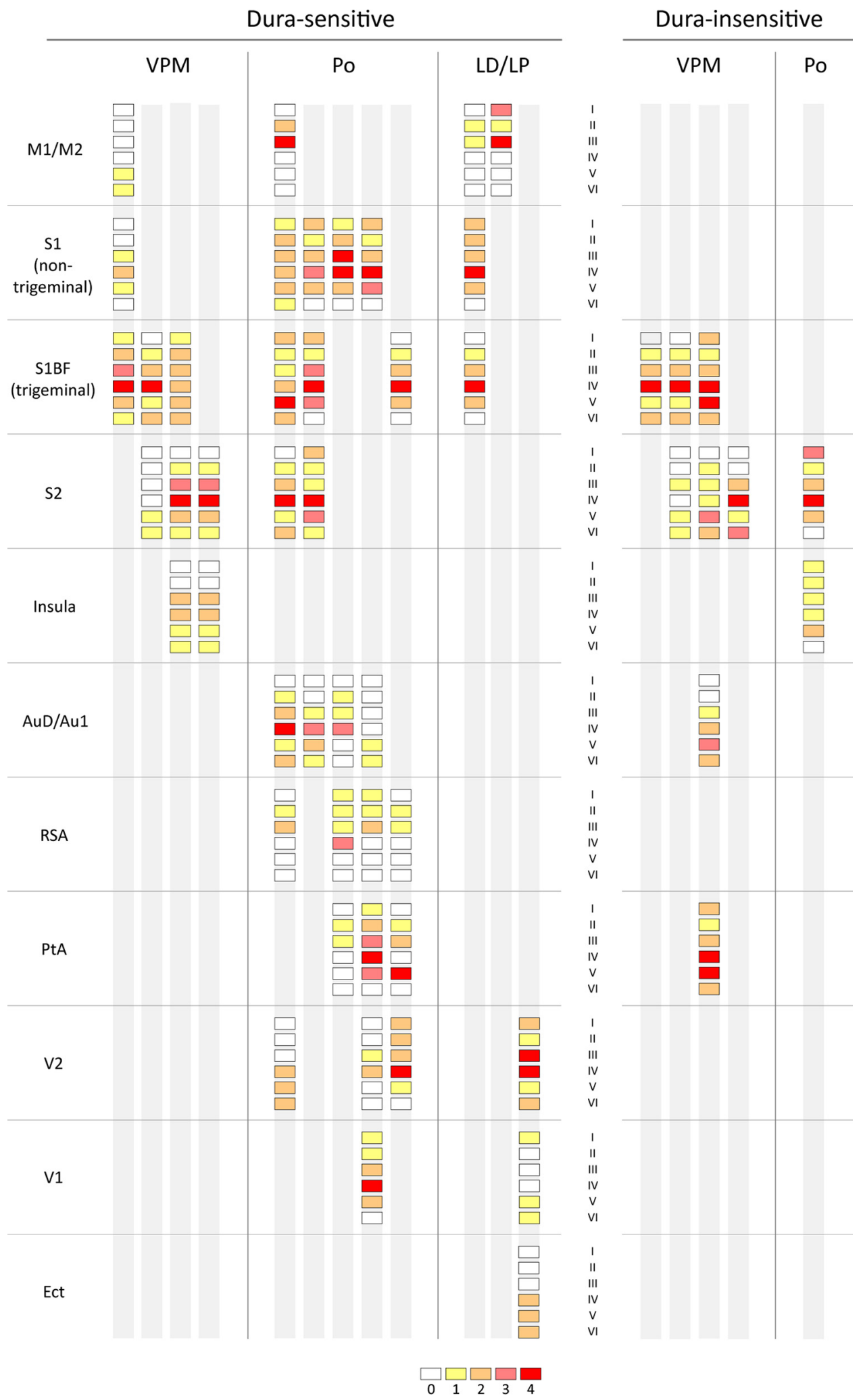

Figure 9. Relative laminar density of terminal arbors of dura-sensitive and dura-insensitive thalamocortical neurons. Fiber density ( $0-4)$ is color coded according to the scale at the bottom.

high density were observed in all layers of V1 and V2 overall, but the lamination pattern varied greatly from one neuron to the next (Fig. 9).

\section{Discussion}

This study unveiled the final thalamocortical limb of a pain pathway that is thought to underlie migraine headache. Most signifi- cant was the finding that each trigeminovascular neuron sampled in higher-order relay thalamic nuclei (Po, LD, LP) projected in multiple directions into anatomically distinct cortical areas involved in functions as diverse as regulation of affect, motor capacity, visual and auditory perception, spatial orientation, memory retrieval, and olfaction. Furthermore, trigeminovascu- 


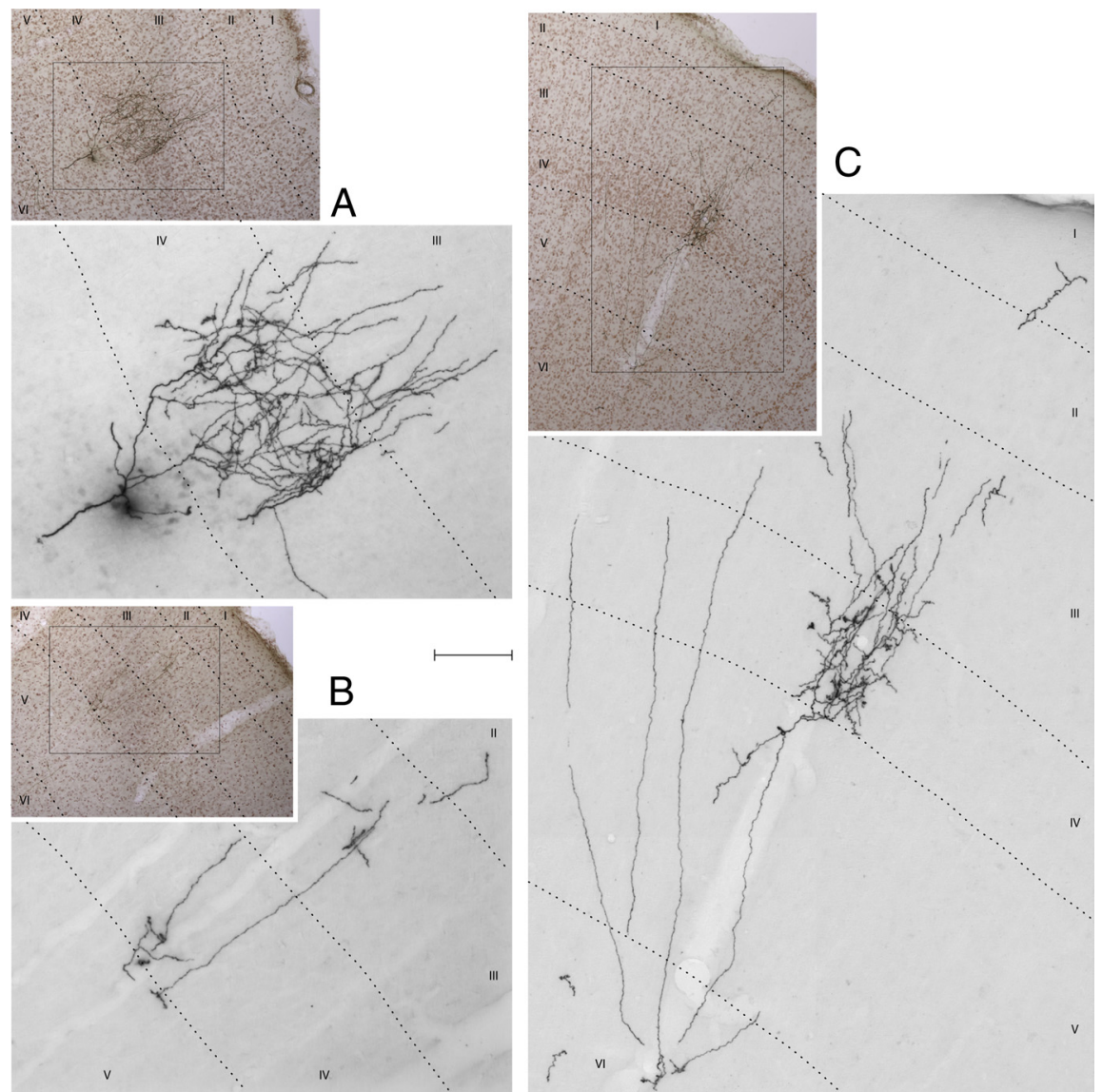

Figure 10. Photomicrographs of axonal arbors in the trigeminal S1 area (S1BF). $\boldsymbol{A}$, Dura-insensitive VPM neuron. $\boldsymbol{B}, \boldsymbol{C}$, Durasensitive VPM neurons. Roman numerals mark the cortical layers. Immunostained fibers were captured before (monochrome images) and after counterstaining of the tissue with neutral red (color images). Boxed areas in the color images correspond to the higher-power monochrome images. Scale bar, 300 and $100 \mu \mathrm{m}$ for color and monochrome images, respectively.

lar neurons of the Po (a higher-order relay nucleus) projected well beyond the so-called "pain matrix" areas (S1, S2, insula), which were the main, if not exclusive, termination territory of their counterpart neurons in VPM (a first-order relay nucleus). This diverse pattern of connections arising from a single axon suggests that trigeminovascular neurons of higher-order relay thalamic nuclei are wired-ready to disseminate information to many cortical areas simultaneously and directly, which stands to explain the diversity of neurological disturbances associated with migraine headache. This pattern of direct projections to multiple cortices is distinct from the indirect, back-and-forth thalamocorticothalamic crosstalk that is mediated specifically by pyramidal cells in layer 5 of various cortical areas (Sherman, 2005; Jones, 2009).

Because the discussion of the extent of thalamocortical projections from each of the studied thalamic nuclei was based on a relatively small number of neurons, we cannot rule out the possibility that other neurons in the studied nuclei project to cortical areas not described here.

Dura-sensitive neurons have been identified previously in the thalamic VPM, Po, and LP/LD nuclei (Davis and Dostrovsky, 1988b; Zagami and Lambert, 1990; Angus-Leppan et al., 1995; Shields and Goadsby, 2005; Burstein et al., 2010; Noseda et al., 2010). Based on the current study, we propose that dura-sensitive neurons in VPM, Po, and LP/LD are capable of mediating different aspects of migraine headache and that their different roles are determined by their distinct pattern of projections in the cortex
(Fig. 14). For example, first-order relay dura-sensitive neurons in VPM project mainly to components of the so-called "pain matrix" (trigeminal S1, S2, and the insular cortex) and thus are more likely to play a role in the perception of the headache (i.e., location, intensity, and quality), whereas dense projections of individual higher-order relay dura-sensitive neurons in Po to non-trigeminal areas of $\mathrm{S} 1$, as well as auditory, visual, retrosplenial, ectorhinal, and parietal association cortices may place them in a position to contribute more significantly to other aspects of the "migraine experience," which include disturbances in neurological functions involved in vision, auditory, memory, motor, and cognitive performance and perhaps extended allodynia. In the context of thalamocorticothalamic processing, such interconnectivity may be considered as running in parallel to the well-defined zig-zag-like circuit in which higher-order neurons relay sensory information from one cortical area to another through pyramidal cells in cortical layer 5 (Sherman, 2005; Jones, 2009).

Heavy projections of dura-sensitive neurons in all studied thalamic nuclei to the trigeminal area of S1, which follows the principles of thalamocortical projections of sensory thalamic neurons (Meyer et al., 2010), suggest that they can also constitute a redundant network that ensures that critical information will be relayed from the thalamus to S1 and S2, despite the extensive modulation to which they are subjected by neurons in layer 6 of the cortex and the reticular thalamic nucleus (Sherman, 2005; Jones, 2009).

Subcortical axonal trajectories of dura-sensitive VPM neurons differ from those of their counterparts in Po and LP/LD because they were the only one to issue terminal arborization in the basal ganglia. Although we have no functional explanation for this selectivity, the findings may explain the altered processing seen in this area during migraine (Afridi et al., 2005) and cluster headache (May et al., 2000).

Based on imaging studies of somatic pain (Coghill et al., 1994; Becerra et al., 1999; Tölle et al., 1999; Peyron et al., 2000; Bingel et al., 2003), we propose that the ability of migraineurs to perceive the source, severity, and characteristics of headache involve nociceptive signals that reach the trigeminal area of S1, S2, and insula. S2 and the posterior insula have been implicated in encoding thermal pain intensity (Peyron et al., 1999; Zhang et al., 1999), which is manifested as thermal allodynia during migraine (Burstein et al., 2000). Moreover, evidence for amplification of activity in the thalamus, S2, and the posterior insular cortex after the provocation of pain by innocuous stimulation of the skin (Peyron et al., 1998) are in agreement with our proposal that sensitization of trigeminovascular thalamic neurons projecting to S2 and insula promotes extended allodynia during migraine (Burstein et al., 2010).

The anterior insula has been considered critical for normal processing of pain affect (Phillips et al., 1997; Büchel et al., 1999), 
and its lesion produced pain asymbolia, a condition in which individuals exhibit proper pain sensation but inappropriate pain affect (Berthier et al., 1988). This concept has been challenged recently in a study in which individuals with lesions of the insula (Starr et al., 2009) exhibited higher pain intensity rating and elevated pain-related activation of the primary somatosensory cortex, raising the possibility that the insula uses previously acquired cognitive information to modulate the activity of other cortical areas that constitute the "pain matrix" (Treede et al., 2000). In the context of migraine, projections of dura-sensitive thalamic neurons to the anterior insula, if present in humans, may play a role in fine-tuning incoming pain signals into other cortical areas involved in pain processing (i.e., S1, S2, prefrontal, anterior cingulate, parahippocampal gyrus) by using cognitive information gained through previous experience with this recurrent pain disorder.

Another group of cortical areas that receive direct input from thalamic durasensitive neurons are members of a network of brain regions that support a range of cognitive functions that include spatial memory, navigation in space, imagination, and planning for the future (Torrealba and Valdes, 2008; Vann et al., 2009). Of particular interest was the dense labeling in the PtA and RSA. The rat PtA (analogous to Brodmann areas 5 and 7) is thought to be involved in tasks that require association between different sensory modalities (Torrealba and Valdes, 2008). Functionally, the PtA is thought to play a role in determining where objects are in relation to parts of the body, which is critical for proper ability to locate objects in space. In the context of migraine, input to these areas from dura-sensitive neurons may explain what appears to patients as motor clumsiness and difficulty in focusing attention on particular tasks in a complex environment with multiple distractions. The RSA (analogous to Brodmann areas 29 and 30 ) is considered essential for acquiring new information, retrieval of short-term autobiographical events, successful navigation through familiar environment, and speech production and comprehension (Valenstein et al., 1987; Maguire, 2001; Awad et al., 2007; Vann et al., 2009). In the context of migraine, input to the RSA from dura-sensitive neurons, if exists in the human, may contribute to the transient decline in speech production and comprehension, as well as cognitive functions.

Under normal conditions, the motor cortex exhibits $20 \mathrm{~Hz}$ oscillatory activity that is essential for setting up proper levels of intracortical inhibition and inhibition of thalamic relay neurons (Peyron et al., 1995; García-Larrea et al., 1999). Because this oscillatory activity is disrupted in the presence of chronic or evoked pain (Juottonen et al., 2002; Raij et al., 2004; Lefaucheur, 2006), it is tempting to propose that projections of dura-sensitive thalamic neurons to the primary motor cortex may promote cortical hyperexcitability in migraineurs. Critical to future understanding of migraine pathophysiology is whether attack frequency and associated symptoms are results of inherent defect in motor cortex ability to govern cortical excitability and activity of descending modulatory pain pathways in the brainstem or a breakdown of oscillatory activity after repeated bombardment of its neurons by the noxious inputs it receives during migraine.

Given our recent description of a direct retino-thalamocortical pathway for exacerbation of migraine by light (Noseda et al., 2010), it may not be surprising that dura-sensitive neurons in higher-order relay nuclei project heavily to all layers of the visual (as well as other) cortices. Although speculative, enhanced firing in these neurons attributable to nociceptive input from the dura may interfere with proper cortical processing of photic signals. In the context of migraine, such disruption may mediate the perception of blurred or double vision, saturation of colors, and the abnormal intolerance to light (Liveing, 1873). Similarly, projections of these dura-sensitive neurons to the auditory cortices may alter proper cortical processing of auditory signals, resulting in extreme intolerance to noise.

Dura-sensitive neurons in VPM and Po issued terminal arborization in all cortical layers of the somatosensory and visual cortices and in almost all layers of the other cortical areas to which they project. The absence of terminal arborization in layer 

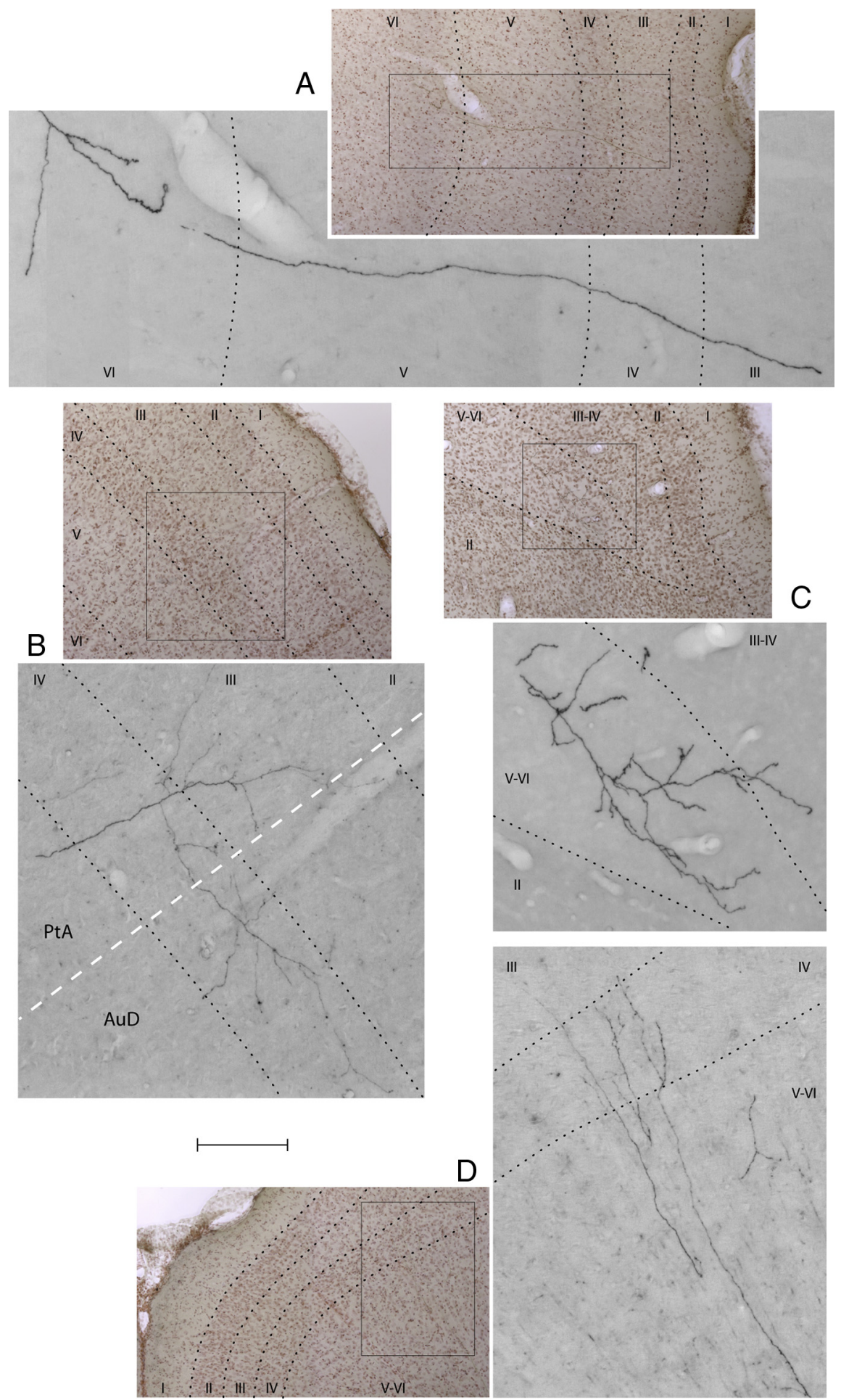

Figure 12. Photomicrographs of axonal arbors in insular, auditory, parietal, ectorhinal, and motor cortices. $\boldsymbol{A}$, Insula, durasensitive VPM neuron. B, PtA and AuD, dura-sensitive Po neuron. C, Ectorhinal, dura-sensitive LP neuron. D, M1/M2, dura-sensitive LD neuron. For additional information, including cortical layers and scales, see Figure 10.

6 of the RSA, PtA, and the motor cortex may be significant in migraine pathophysiology if holds true in future studies of a much greater scale. Given the input to all layers, it seems reasonable to propose that activation of dura-sensitive thalamic neurons during migraine can set off localized and intrahemispheric communication needed for modulation of pyramidal cells in layers 5 and 6, which, respectively, regulate cranial nerves and sensory and motor spinal cord neurons and, most importantly, the thalamic input to the cortex.

We cannot conclude with certainty that the widespread thalamocortical projections are unique to dura-sensitive neurons. However, in view of the limited cortical projections of nociceptive thalamic neurons that respond to somatic skin stimulation (Gauriau and Bernard, 2004; Monconduit and Villanueva, 2005; Monconduit et al., 2006), we suggest that the projection pattern of dura-sensitive neurons may be a distinct feature of the trigeminovascular pathway.

\section{References}

Afridi SK, Matharu MS, Lee L, Kaube H, Friston KJ, Frackowiak RS, Goadsby PJ (2005) A PET study exploring the laterality of brainstem activation in migraine using glyceryl trinitrate. Brain 128:932-939.

Angus-Leppan H, Olausson B, Boers P, Lambert GA (1995) Convergence of afferents from superior sagittal sinus and tooth pulp on cells in the thalamus of the cat. Cephalalgia 15:191-199.

Angus-Leppan H, Lambert GA, Michalicek J (1997) Convergence of occipital nerve and superior sagittal sinus input in the cervical spinal cord of the cat. Cephalalgia 17:625-630, discussion 623

Awad M, Warren JE, Scott SK, Turkheimer FE, Wise RJ (2007) A common system for the comprehension and production of narrative speech. J Neurosci 27:11455-11464.

Becerra LR, Breiter HC, Stojanovic M, Fishman S, Edwards A, Comite AR, Gonzalez RG, Borsook D (1999) Human brain activation under controlled thermal stimulation and habituation to noxious heat: an fMRI study. Magn Reson Med 41:1044-1057.

Berthier M, Starkstein S, Leiguarda R (1988) Asymbolia for pain: a sensory-limbic disconnection syndrome. Ann Neurol 24:41-49.

Bingel U, Quante M, Knab R, Bromm B, Weiller C, Büchel C (2003) Single trial fMRI reveals significant contralateral bias in responses to laser pain within thalamus and somatosensory cortices. Neuroimage 18: $740-748$.

Büchel C, Dolan RJ, Armony JL, Friston KJ (1999) Amygdala-hippocampal involvement in human aversive trace conditioning revealed through event-related functional magnetic resonance imaging. J Neurosci 19:1086910876.

Burstein R, Yamamura H, Malick A, Strassman AM (1998) Chemical stimulation of the intracranial dura induces enhanced responses to facial stimulation in brain stem trigeminal neurons. J Neurophysiol 79:964-982.

Burstein R, Yarnitsky D, Goor-Aryeh I, Ransil BJ, Bajwa ZH (2000) An association between migraine and cutaneous allodynia. Ann Neu-

rol 47:614-624.

Burstein R, Jakubowski M, Garcia-Nicas E, Kainz V, Bajwa Z, Hargreaves R, Becerra L, Borsook D (2010) Thalamic sensitization transforms localized pain into widespread allodynia. Ann Neurol 68:81-91.

Coghill RC, Talbot JD, Evans AC, Meyer E, Gjedde A, Bushnell MC, Duncan GH (1994) Distributed processing of pain and vibration by the human brain. J Neurosci 14:4095-4108. 

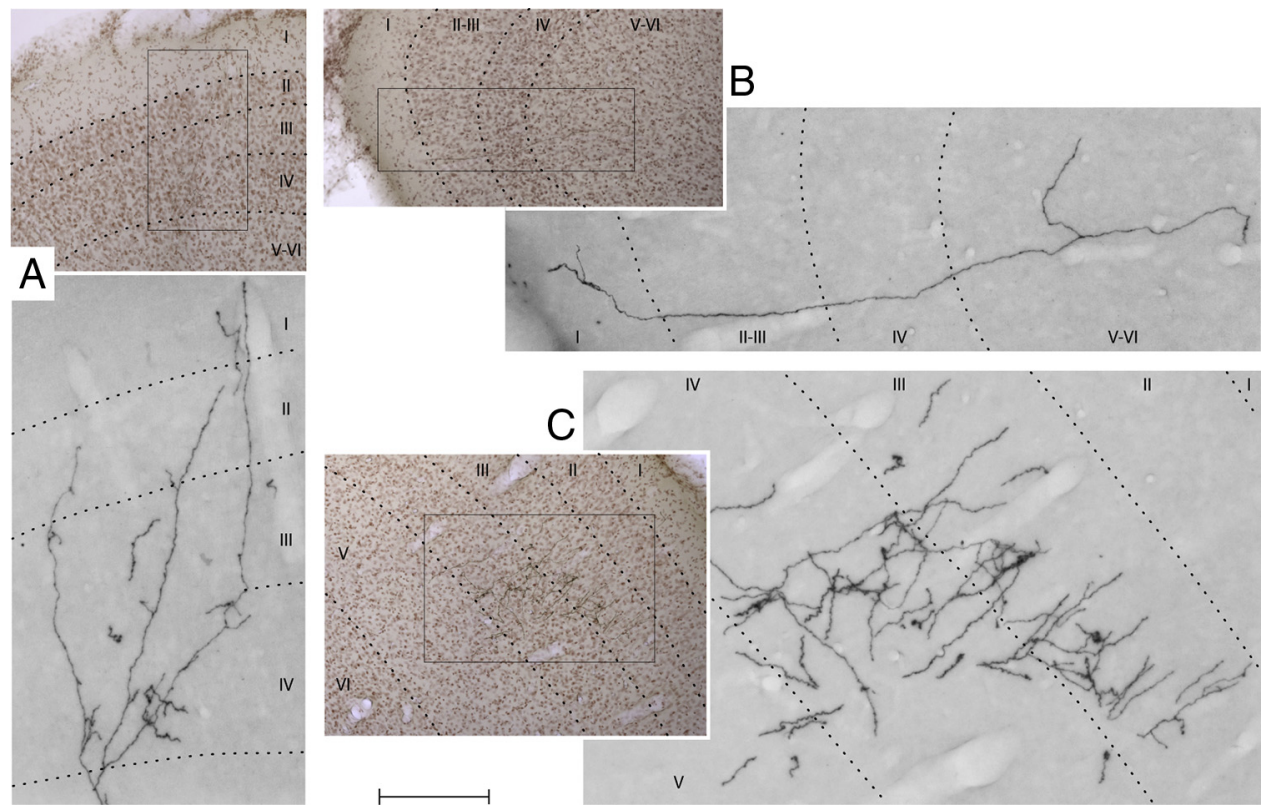

Figure 13. Photomicrographs of axonal arbors in the visual cortices of a dura-sensitive LP neuron. $A$, V2MM. $\boldsymbol{B}$, V1M. $C$, V2L. For additional information, including cortical layers and scales, see Figure 10.

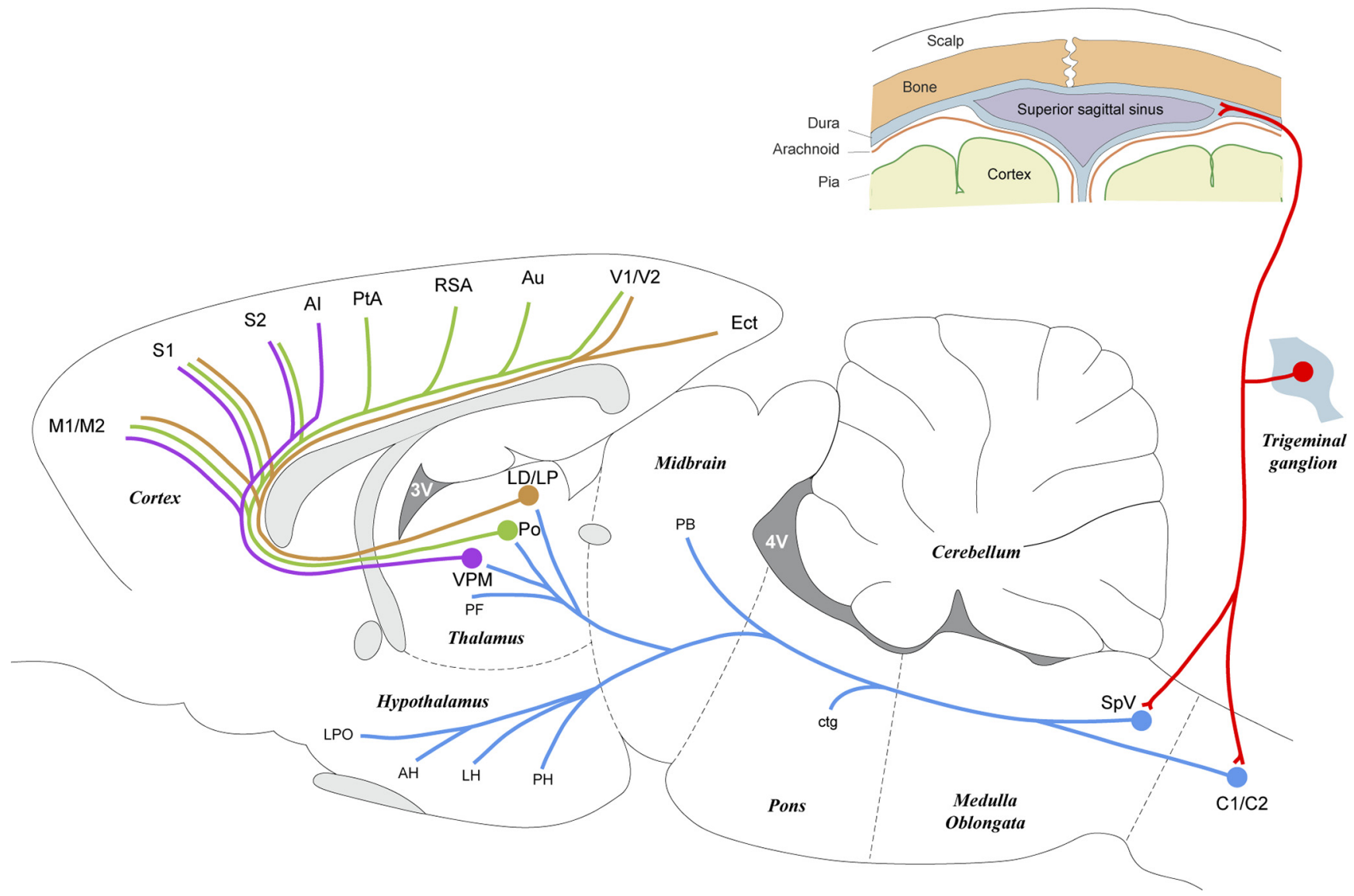

Figure 14. Schematic illustration of the trigeminovascular pathway between the meninges and the cortex. The peripheral limb (meningeal nociceptors) is shown in red. The ascending limb from the spinal trigeminal nucleus (Vc and C1/C2) to the brainstem, thalamus, and hypothalamus is shown in blue. The thalamocortical projections from VPM, PO, and LD/LP are shown in purple, green, and orange, respectively. AH, Anterior hypothalamus; ctg, central tegmental area; LH, lateral hypothalamus; LPO, lateral posterior area; PB, parabrachial area; PH, posterior hypothalamus. For additional abbreviations, see previous figure legends. 
Davis KD, Dostrovsky JO (1988a) Responses of feline trigeminal spinal tract nucleus neurons to stimulation of the middle meningeal artery and sagittal sinus. J Neurophysiol 59:648-666.

Davis KD, Dostrovsky JO (1988b) Properties of feline thalamic neurons activated by stimulation of the middle meningeal artery and sagittal sinus. Brain Res 454:89-100.

DeFelipe J, Alonso-Nanclares L, Arellano JI (2002) Microstructure of the neocortex: comparative aspects. J Neurocytol 31:299-316.

Ebersberger A, Ringkamp M, Reeh PW, HandwerkerHO (1997) Recordings from brain stem neurons responding to chemical stimulation of the subarachnoid space. J Neurophysiol 77:3122-3133.

García-Larrea L, Peyron R, Mertens P, Gregoire MC, Lavenne F, Le Bars D, Convers P, Mauguière F, Sindou M, Laurent B (1999) Electrical stimulation of motor cortex for pain control: a combined PET-scan and electrophysiological study. Pain 83:259-273.

Gauriau C, Bernard JF (2004) Posterior triangular thalamic neurons convey nociceptive messages to the secondary somatosensory and insular cortices in the rat. J Neurosci 24:752-761.

Groh A, Meyer HS, Schmidt EF, Heintz N, Sakmann B, Krieger P (2010) Celltype specific properties of pyramidal neurons in neocortex underlying a layout that is modifiable depending on the cortical area. Cereb Cortex $20: 826-836$

Jones EG (2009) Synchrony in the interconnected circuitry of the thalamus and cerebral cortex. Ann N Y Acad Sci 1157:10-23.

Juottonen K, Gockel M, Silén T, Hurri H, Hari R, Forss N (2002) Altered central sensorimotor processing in patients with complex regional pain syndrome. Pain 98:315-323.

Kaube H, Hoskin KL, Goadsby PJ (1992) Activation of the trigeminovascular system by mechanical distension of the superior sagittal sinus in the cat. Cephalalgia 12:133-136.

Lefaucheur JP (2006) The use of repetitive transcranial magnetic stimulation (rTMS) in chronic neuropathic pain. Neurophysiol Clin 36:117-124.

Levy D, Strassman AM (2002) Mechanical response properties of A and C primary afferent neurons innervating the rat intracranial dura. J Neurophysiol 88:3021-3031.

Liu Y, Broman J, Edvinsson L (2004) Central projections of sensory innervation of the rat superior sagittal sinus. Neuroscience 129:431-437.

Liu Y, Broman J, Edvinsson L (2008) Central projections of the sensory innervation of the rat middle meningeal artery. Brain Res 1208:103-110.

Liveing E (1873) On megrim, sick headache. Nijmegen, The Netherlands: Arts and Boeve Publishers.

Maguire EA (2001) The retrosplenial contribution to human navigation: a review of lesion and neuroimaging findings. Scand J Psychol 42:225-238.

May A, Bahra A, Büchel C, Frackowiak RS, Goadsby PJ (2000) PET and MRA findings in cluster headache and MRA in experimental pain. Neurology 55:1328-1335.

Mayberg M, Langer RS, Zervas NT, Moskowitz MA (1981) Perivascular meningeal projections from cat trigeminal ganglia: possible pathway for vascular headaches in man. Science 213:228-230.

Mayberg MR, Zervas NT, Moskowitz MA (1984) Trigeminal projections to supratentorial pial and dural blood vessels in cats demonstrated by horseradish peroxidase histochemistry. J Comp Neurol 223:46-56.

Meyer HS, Wimmer VC, Hemberger M, Bruno RM, de Kock CP, Frick A, Sakmann B, Helmstaedter M (2010) Cell type-specific thalamic innervation in a column of rat vibrissal cortex. Cereb Cortex 20:2287-2303.

Monconduit L, Villanueva L (2005) The lateral ventromedial thalamic nucleus spreads nociceptive signals from the whole body surface to layer I of the frontal cortex. Eur J Neurosci 21:3395-3402.

Monconduit L, Lopez-Avila A, Molat JL, Chalus M, Villanueva L (2006) Corticofugal output from the primary somatosensory cortex selectively modulates innocuous and noxious inputs in the rat spinothalamic system. J Neurosci 26:8441-8450.

Noseda R, Kainz V, Jakubowski M, Gooley JJ, Saper CB, Digre K, Burstein R (2010) A neural mechanism for exacerbation of headache by light. Nat Neurosci 13:239-245.

Paxinos G, Watson C (1998) The rat brain in stereotaxic coordinates, Ed 4. Orlando, FL: Academic.
Peyron R, Garcia-Larrea L, Deiber MP, Cinotti L, Convers P, Sindou M, Mauguière F, Laurent B (1995) Electrical stimulation of precentral cortical area in the treatment of central pain: electrophysiological and PET study. Pain 62:275-286.

Peyron R, García-Larrea L, Grégoire MC, Convers P, Lavenne F, Veyre L, Froment JC, Mauguière F, Michel D, Laurent B (1998) Allodynia after lateral-medullary (Wallenberg) infarct. A PET study. Brain 121:345-356.

Peyron R, García-Larrea L, Grégoire MC, Costes N, Convers P, Lavenne F, Mauguière F, Michel D, Laurent B (1999) Haemodynamic brain responses to acute pain in humans: sensory and attentional networks. Brain 122:1765-1780.

Peyron R, Laurent B, García-Larrea L (2000) Functional imaging of brain responses to pain. A review and meta-analysis (2000). Neurophysiol Clin 30:263-288.

Phillips ML, Young AW, Senior C, Brammer M, Andrew C, Calder AJ, Bullmore ET, Perrett DI, Rowland D, Williams SC, Gray JA, David AS (1997) A specific neural substrate for perceiving facial expressions of disgust. Nature 389:495-498.

Pinault D (1996) A novel single-cell staining procedure performed in vivo under electrophysiological control: morpho-functional features of juxtacellularly labeled thalamic cells and other central neurons with biocytin or neurobiotin. J Neurosci Methods 65:113-136.

Raij TT, Forss N, Stancák A, Hari R (2004) Modulation of motor-cortex oscillatory activity by painful Adelta- and C-fiber stimuli. Neuroimage 23:569-573.

Sherman SM (2005) Thalamic relays and cortical functioning. Prog Brain Res 149:107-126.

Shields KG, Goadsby PJ (2005) Propranolol modulates trigeminovascular responses in thalamic ventroposteromedial nucleus: a role in migraine? Brain 128:86-97.

Shipp S (2007) Structure and function of the cerebral cortex. Curr Biol 17:R443-R449.

Starr CJ, Sawaki L, Wittenberg GF, Burdette JH, Oshiro Y, Quevedo AS, Coghill RC (2009) Roles of the insular cortex in the modulation of pain: insights from brain lesions. J Neurosci 29:2684-2694.

Strassman AM, Mineta Y, Vos BP (1994) Distribution of fos-like immunoreactivity in the medullary and upper cervical dorsal horn produced by stimulation of dural blood vessels in the rat. J Neurosci 14:3725-3735.

Strassman AM, Raymond SA, Burstein R (1996) Sensitization of meningeal sensory neurons and the origin of headaches. Nature 384:560-564.

Tölle TR, Kaufmann T, Siessmeier T, Lautenbacher S, Berthele A, Munz F, Zieglgänsberger W, Willoch F, Schwaiger M, Conrad B, Bartenstein P (1999) Region-specific encoding of sensory and affective components of pain in the human brain: a positron emission tomography correlation analysis. Ann Neurol 45:40-47.

Torrealba F, Valdés JL (2008) The parietal association cortex of the rat. Biol Res 41: 369-377.

Treede RD, Apkarian AV, Bromm B, Greenspan JD, Lenz FA (2000) Cortical representation of pain: functional characterization of nociceptive areas near the lateral sulcus. Pain 87:113-119.

Valenstein E, Bowers D, Verfaellie M, Heilman KM, Day A, Watson RT (1987) Retrosplenial amnesia. Brain 110:1631-1646.

Vann SD, Aggleton JP, Maguire EA (2009) What does the retrosplenial cortex do? Nat Rev Neurosci 10:792-802.

Vogt BA, Peters A (1981) Form and distribution of neurons in rat cingulate cortex: areas 32, 24, and 29. J Comp Neurol 195:603-625.

Yamamura H, Malick A, Chamberlin NL, Burstein R (1999) Cardiovascular and neuronal responses to head stimulation reflect central sensitization and cutaneous allodynia in a rat model of migraine. J Neurophysiol 81:479-493.

Zagami AS, Lambert GA (1990) Stimulation of cranial vessels excites nociceptive neurones in several thalamic nuclei of the cat. Exp Brain Res 81:552-566.

Zagami AS, Lambert GA (1991) Craniovascular application of capsaicin activates nociceptive thalamic neurones in the cat. Neurosci Lett 121:187-190

Zhang ZH, Dougherty PM, Oppenheimer SM (1999) Monkey insular cortex neurons respond to baroreceptive and somatosensory convergent inputs. Neuroscience 94:351-360. 